\title{
CONCEPTS
}

\section{How To Teach Emergency Procedural Skills in an Outdoor Environment Using Low-Fidelity Simulation}

\author{
Kathleen D. Saxon, MD; Alison P.R. Kapadia, MD; Nadia S. Juneja, MD; Benjamin S. Bassin, MD \\ From the Department of Emergency Medicine, University of Michigan, Ann Arbor, MI.
}

\begin{abstract}
Teaching emergency procedural skills in a wilderness setting can be logistically challenging. To teach these skills as part of a wilderness medicine elective for medical students, we designed an outdoor simulation session with low-fidelity models. The session involved 6 stations in which procedural skills were taught using homemade low-fidelity simulators. At each station, the students encountered a "victim," who required an emergency procedure that was performed using the low-fidelity model. The models are easy and inexpensive to construct, and their design and implementation in the session is described here. Using low-fidelity simulation models in an outdoor setting is an effective teaching tool for emergency wilderness medicine procedures and can easily be reproduced in future wilderness medicine courses.
\end{abstract}

Key words: simulation, wilderness medicine education

\section{Introduction}

The use of high-fidelity simulation technology has been well established and supported in medical education. To teach traditional medical scenarios, the simulated hospital setting where these models are used can easily translate to the real-life experience. However, by the very nature of teaching the principles of wilderness medicine, it is difficult to emulate a similar teaching environment outdoors. Unique circumstances such as terrain and environmental factors cannot be easily re-created. A prior study has demonstrated success with high-fidelity models in an outdoor setting. ${ }^{1}$ Although high-fidelity models do provide advantages such as realistic physical examination findings and programmable sequences, they come with significant cost. Because of the expense, they may not be ideal for use in an outdoor scenario where damage from handling against rough terrain or weather could cause irreparable damage. Yet, the ability to practice high-risk procedures or scenarios in these settings is desirable. To meet such a need, we designed low-fidelity and low-cost simulators to use in a wilderness setting. We then used these models during an outdoor simulation session to teach emergency procedural skills as part of a wilderness

Corresponding author: Kathleen Saxon, MD, Colorado Permanente Medical Group, 1835 N Franklin Street, Denver, CO 80210 (e-mail: kdsaxon@gmail.com). medicine elective for medical students. This simulation session is easily reproducible and low-cost, and offers an excellent method for students to obtain clinical experience and hands-on practice.

\section{Methods \\ OVERVIEW OF THE SIMULATION SESSION}

We designed an outdoor simulation laboratory session with 13 fourth-year medical students as part of a monthlong elective in wilderness medicine. Three emergency medicine residents organized the session, and materials were provided by our Department of Emergency Medicine. Before the session, the students had received a lecture and demonstrations to learn the procedures. The session was held outdoors in a large arboretum and included 6 stations in which emergency procedural skills were taught using homemade low-fidelity simulators. The students located each station by orienteering with a map and compass. On arrival at each station, a "victim" was encountered, who was acting as the patient in the simulation scenario. The students were required to rapidly assess the scene and the patient and provide appropriate care and stabilization. Each victim required an emergency procedure, and some of these were performed using a low-fidelity model. After the students completed all 6 stations, there was a debriefing session during which 
instructors reviewed the students' performance and discussed indications and appropriateness of performing these measures in a remote environment.

The students were placed in pairs and then competed to finish the session most quickly, and received points for finding each station, appropriately managing the patients, and performing the required procedures. Each pair was required to supply their own first aid kit without prior knowledge of what they would encounter. Points were awarded for the team that was most prepared based on the completeness of their first aid kit. Students had points deducted if they needed to borrow equipment at the station. For example, in performing thoracostomy, some groups had brought their own multitool or knife, as well as tubing that could be used for the chest tube. If they had not brought such items, they were given a multitool and hydration tubing, but points were deducted from their score.

\section{STATION DESIGN}

The procedures at the stations included cricothyroidotomy, thoracostomy, needle decompression, control of epistaxis, lateral canthotomy, and application of a pelvic binder, a femur traction splint, and an occlusive dressing for a sucking chest wound. Each station had a written guide that included a list of necessary equipment, the scenario and scene, and notes for the patient, which included vital signs, relevant history, the AMPLE (Allergies, Medications, Past illnesses, Last meal, Events preceding injury) history, and physical examination findings. In addition, at each station, critical actions were required, including identifying the diagnosis and performing an appropriate emergency procedure. Based on these key actions, a score was tallied for the performance at the station. Points were awarded for checking for scene safety, wearing gloves or protective gear, and washing hands; obtaining the primary survey, AMPLE history, secondary survey, and making the diagnosis; and performing the procedure.

\section{REQUIRED EQUIPMENT}

To perform the session, a large outdoor space is needed in which it is easy to create an orienteering course. Natural landmarks can be used as the station sites, and a topographic map with controls can be given to the students to use. Six moderators are required, as each station needs at least 1 moderator, and 6 prototypes of each model are required, as a new model is provided for each pair of students. The materials used to create each model are described below.

\section{CRICOTHYROIDOTOMY STATION}

At the cricothyroidotomy station, the students encountered a patient at the side of a river. The patient had been camping along the river in a canyon and a boulder had fallen onto their tent, resulting in significant facial trauma. After hearing the scenario, the students examined the moderator at the station, who had makeup indicating blood in the oropharynx, was unable to speak, and had stridor. Once the students diagnosed an upper airway obstruction requiring cricothyroidotomy, they were given the model, which was made of a nebulizer tubing. The tubing rings simulated the tracheal rings, and athletic tape was placed over a hole in the tubing to mimic the cricothyroid membrane. Two packets of ketchup were placed on either side to simulate the surrounding vasculature. Over this, layers of gauze were placed to simulate subcutaneous tissue, and then foam tape was used as skin (Figure 1). Using a multitool, the students cut through the skin to access the membrane, and then used hydration-like tubing to supply ventilation (Figure 2).

\section{NEEDLE DECOMPRESSION AND TUBE THORACOSTOMY STATION}

At the next station, the students came upon a victim who had suffered a 10 -foot fall while free climbing. The victim was wearing a helmet, and on impacting the ground had immediate difficulty breathing. On examination, the students found the moderator holding the

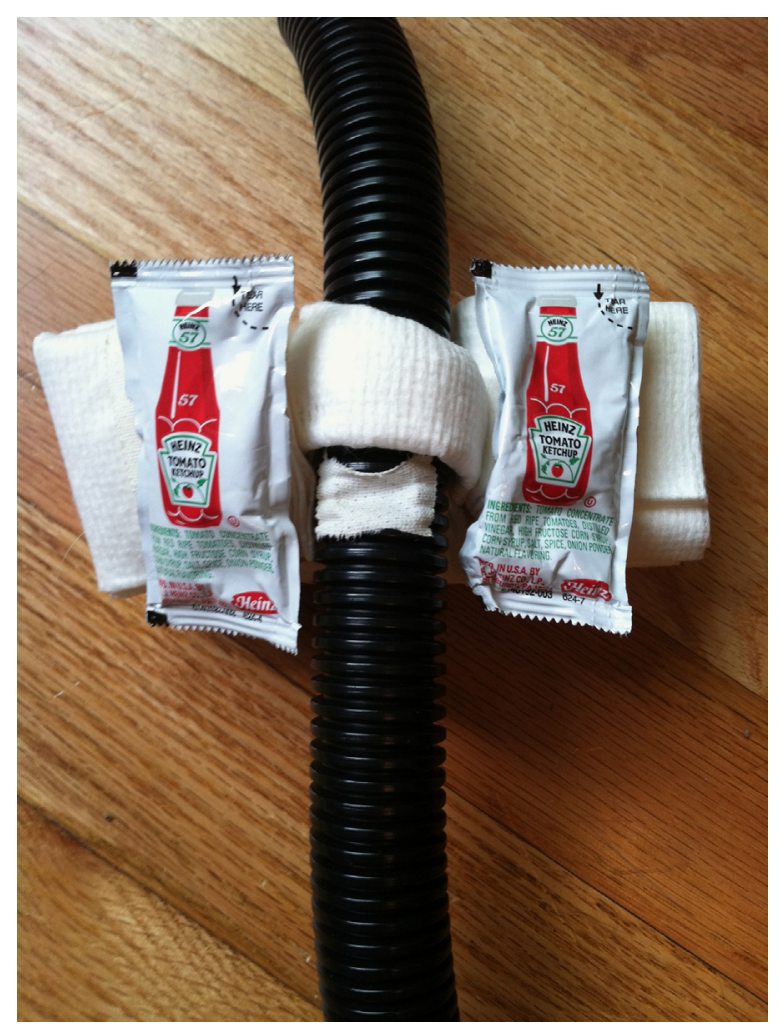

Figure 1. Construction of the cricothyroidotomy model. 


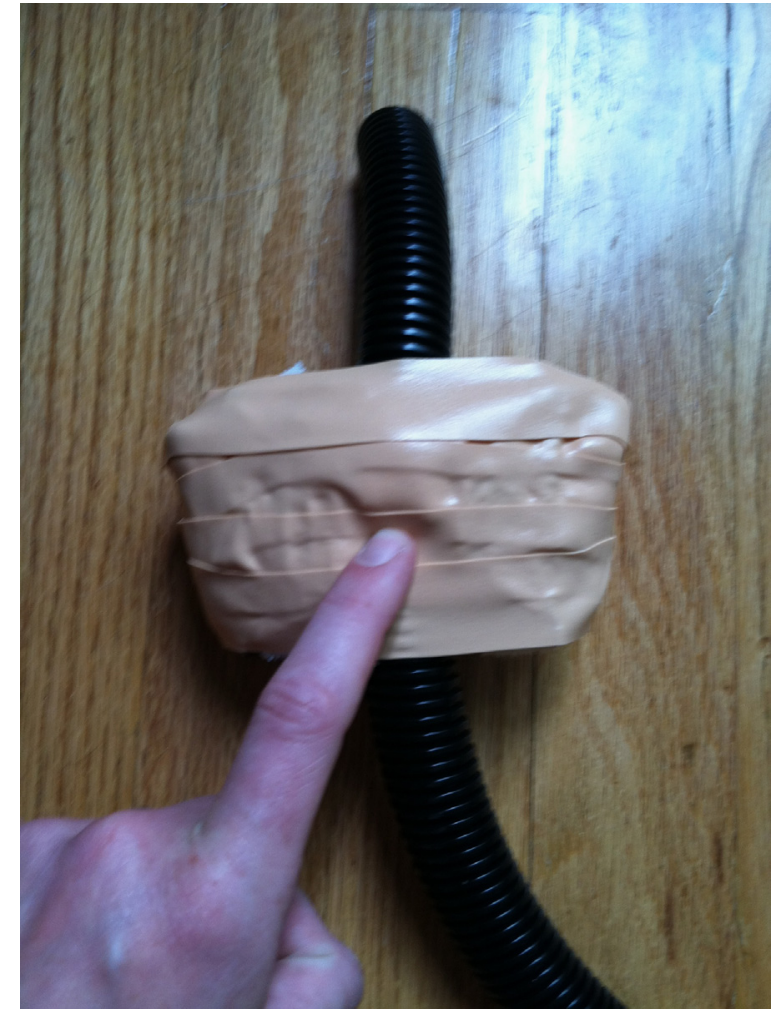

Figure 2. Palpating the cricothyroid membrane on the cricothyroidotomy model.

right side of the chest and speaking in 1- to 2-word phrases. When they examined the chest, they were told there was a lack of breath sounds over the right side and tracheal deviation. When they decided to perform needle decompression, they were given a model. The base was a wooden tangerine box with an inflated balloon inside. An empty shoebox could also be used. Over this, a package of pork ribs was placed. Thickened Jell-O was used as subcutaneous tissue, and foam tape was pulled over the entire model to simulate skin. A line had been drawn to indicate the sternum and clavicle. In performing the procedure, the students had to palpate the ribs and the second intercostal space and insert a large-bore needle to access the pleural space. On doing this, the balloon would pop and air would be released (Figure 3). After needle decompression, if the students recognized thoracostomy was required for definitive management, they were given a second model with lines drawn to indicate the axilla and nipple, and performed the procedure accessing the fifth intercostal space with a multitool. Then they used hydration-like tubing for drainage. A discussion ensued with the moderator of the station regarding a Heimlich flutter valve and appropriate drainage.

\section{LATERAL CANTHOTOMY STATION}

The next patient encountered was an elderly man found facedown after falling while trail running. The patient complained of pain and decreased vision in his right eye, and had makeup indicating significant ecchymosis. Of note, he takes warfarin because of a history of atrial fibrillation. When examined, the students are told the patient has decreased visual acuity in the right eye, periorbital edema, proptosis, and a right afferent pupillary defect. A model was provided when the students elected to do a lateral canthotomy for visual loss owing to a retrobulbar hematoma. This model consisted of a paper plate base with a ping-pong ball to simulate the globe. Eyelids were constructed from foam tape, and the inferior crus of the lateral canthal ligament was created from folded athletic tape at $0.5-\mathrm{cm}$ width. This was placed under the lower eyelid made from the foam tape (Figure 4). During the procedure, a multitool was used to clamp the lateral canthal tissues to relieve globe pressure, and then the inferior crus of the lateral canthal ligament was located and incised (Figure 5).

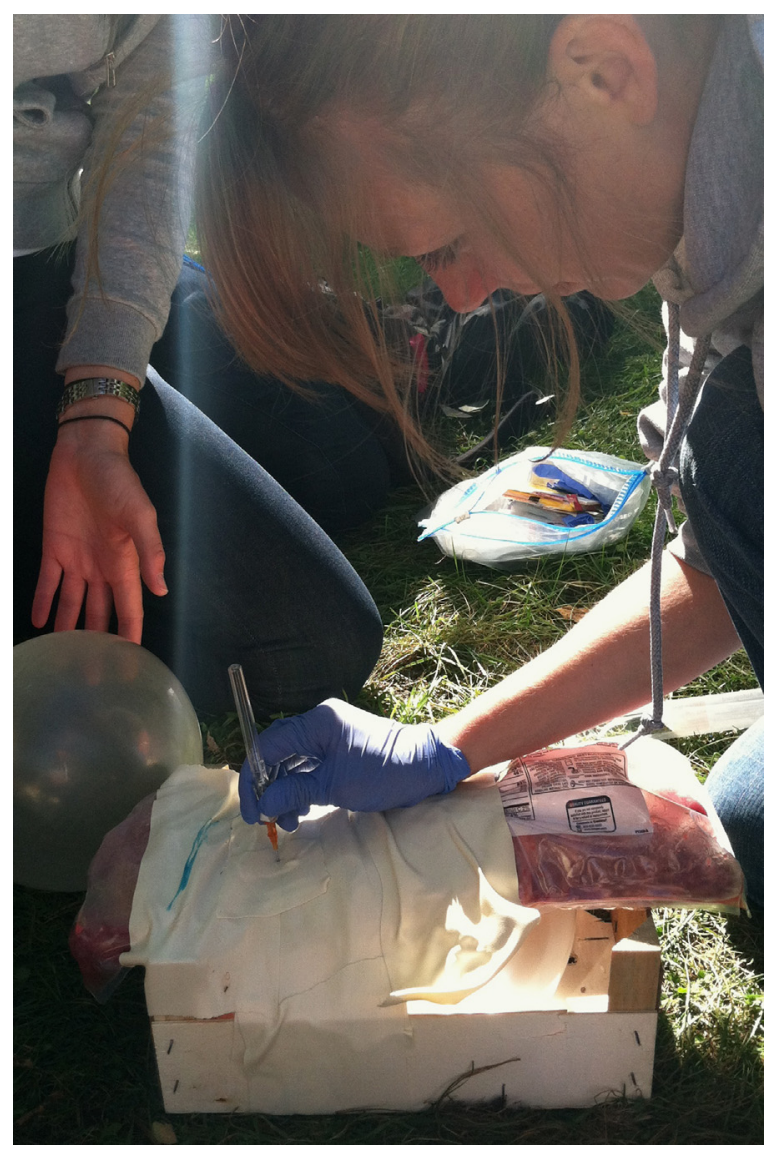

Figure 3. A student performs needle decompression on a low-fidelity model with tape to simulate skin and a slab of ribs encasing an inflated balloon to simulate the pleural cavity. 


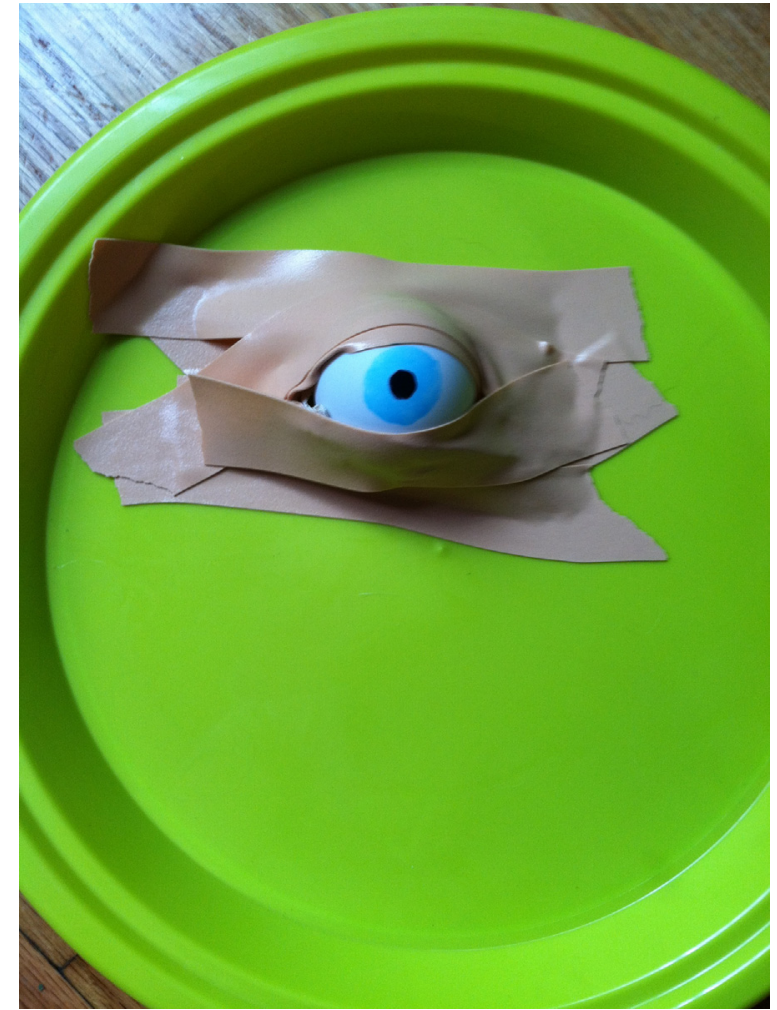

Figure 4. The lateral canthotomy model.

\section{CONTROL OF EPISTAXIS STATION}

At the epistaxis station, the students came upon a patient with a brisk nosebleed after being hit in the face with a crampon while mountaineering. On examination, the moderator wore makeup simulating a nosebleed, and the students were given a model to simulate the bleeding. This model consisted of a potato with a $1-\mathrm{cm}$ diameter hole across the interior. At one entrance to the hole, a 60-mL syringe with fake blood was injected, so that it drained out of the other end, which resembled a bleeding nare. During the procedure, the students identified it was an anterior bleed without a septal hematoma by asking questions, applied pressure for several minutes, and then performed nasal packing with a plan to initiate prophylactic antibiotics. If they needed to borrow materials, they were given a tampon that was soaked in oxymetazoline nasal spray (Figure 6).

\section{FEMUR TRACTION SPLINT STATION}

The last 2 stations included a procedure that did not use low-fidelity models. These were performed using the moderator of the station as the model. At the femur traction splint station, the students found a patient who had just fallen off a horse and was holding his right femur crying in pain. On examination, the right leg was shorter than the left, and the students identified a midshaft femur fracture. They then applied a femur traction splint with 3 anchors and secured this in place. If they required borrowed materials, they were given webbing, a telescoping ski pole, and a mug for the proximal anchor.

\section{PELVIC BINDER AND SUCKING CHEST WOUND STATION}

At the final station, the patient had fallen from a tree and was complaining of severe pain at their left chest and pelvis. The patient was short of breath, speaking in phrases, and holding the left side of their chest. Using the moderator as the model, the students performed an examination and identified severe pelvic tenderness with faint distal lower extremity pulses, and a left open sucking chest wound, which was created with makeup. With a likely unstable pelvic fracture, the students applied a pelvic binder using a shirt or cravats and used a stick as a windlass to increase pressure. With the sucking chest wound, they used materials, such as gauze and tape, to create an occlusive dressing on 4 sides that could be occasionally burped to relieve pressure. Subsequently, a discussion ensued with the moderator regarding the use of

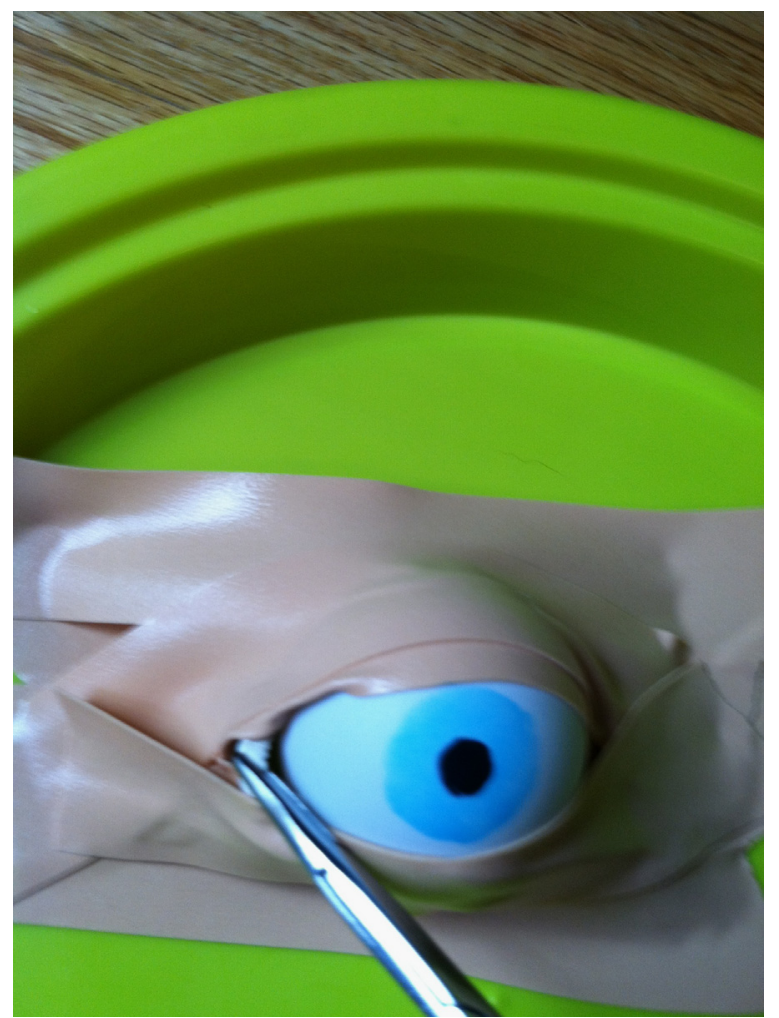

Figure 5. A student crushing the lateral tissues on the lateral canthotomy model. 


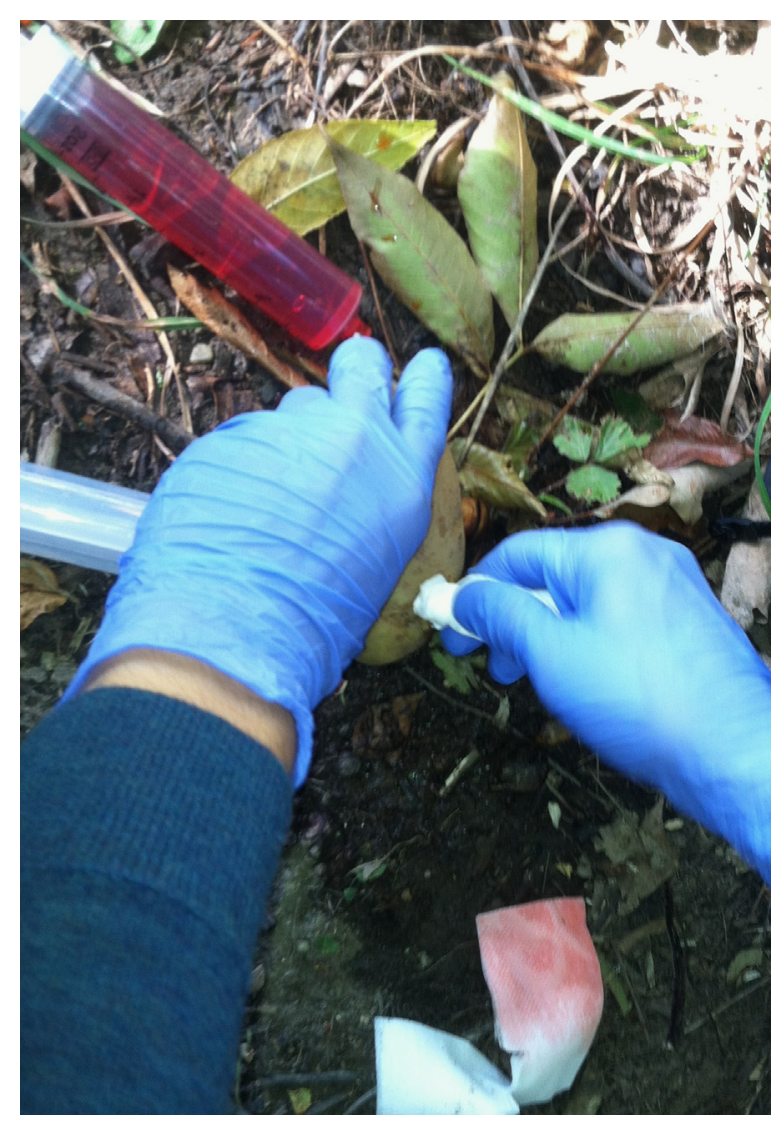

Figure 6. A student controls epistaxis using packing and oxymetazoline hydrochloride on a potato model simulating a bleeding nose.

an Asherman Chest Seal or defibrillator pad for increased efficacy of the occlusive dressing. ${ }^{2}$

\section{EXAMINING THE EFFICACY OF THE SIMULATION SESSION}

To examine the effectiveness of using the low-fidelity models in an outdoor simulation session, a survey was obtained before and after the session. Consent was obtained and exemption was granted by our institutional review board. Before the start of the course, the students answered a short survey regarding comfort levels with the emergency procedures. The scale ranged from 1 (least comfortable) to 5 (most comfortable). In addition, to assess knowledge, each student answered 10 objective questions about how to do the procedure. After the session, the students took the same short survey to rate their comfort levels and answered the same 10 objective questions. The data from each student were paired and analyzed with the Student's $t$ test. With regard to objective knowledge, the students demonstrated improvement with the procedures after the session based on average scores, but only had statistically significant $(P<.05)$ improvement with needle decompression and femur traction splints. There was statistically significant $(P<.05)$ improvement in the students' comfort levels with every procedure performed.

\section{Discussion}

To teach wilderness procedural skills, we used lowfidelity simulation models in an outdoor setting during a fourth-year medical student elective in wilderness medicine. The models were all handmade by the instructors, who were emergency medicine residents, using materials from the grocery or hardware store as well as hospital supply. The models were inexpensive and easy to assemble. This is in stark contrast to highfidelity models available at traditional indoor medical simulation centers.

We found multiple benefits to this simulation model. Students were in groups of 2 and orienteering between stations, thereby making it possible for all students to be simultaneously practicing procedures. The low-fidelity models also allowed for the students to actually perform procedures that are often not possible with a high-fidelity model. For example, students actually cut through simulated cricothyroid membranes, lateral canthi, and the chest wall, and applied a femur traction splint and pelvic binder to a live person. Many high-fidelity simulators do not allow students to actually make incisions. Because of the low cost of the simulators, it is possible to have each pair of students use a new model and actually perform the procedure without concern for preserving an expensive model. Based on the survey data of our session, both objective knowledge as well as comfort with the procedures improved.

In future sessions, there is also room for creativity to come up with new models for other procedures such as tooth reimplantation or reduction of a shoulder dislocation. Although the low-fidelity models offer the opportunity to actually perform the procedure, they are still limited in producing simulated examination findings. For example, in evaluating the victim at each station, the students were still forced to ask questions such as "Do I hear breath sounds on this side of the chest?"

\section{Conclusions}

Using low-fidelity simulation models in an outdoor setting is an effective teaching tool for emergency wilderness medicine procedures and can be easily reproduced during future educational activities, including medical student electives, wilderness medicine courses, and conferences. This model complements the hands-on, active learning style of many adult students. The session 
allowed students to apply their knowledge to the "bedside" and actually practice procedural skills in an austere setting.

\section{Acknowledgments}

Thank you to Henderson McGinnis for inspiring the idea of creating low-fidelity models to teach procedural skills.

\section{References}

1. Lareau SA, Kyzer BD, Hawkins SC, McGinnis HD. Advanced wilderness life support education using hightechnology patient simulation. Wilderness Environ Med. 2010;21:166-170.

2. Rathert N, Gilmore WS. Treating sucking chest wounds and other traumatic chest injuries. J Emerg Med Serv. 2013; August. 